\title{
Genome-wide comparative analysis reveals human-mouse regulatory landscape and evolution
}

Olgert Denas ${ }^{1}$, Richard Sandstrom ${ }^{4}$, Yong Cheng ${ }^{3}$, Kathryn Beal ${ }^{7}$, Javier Herrero ${ }^{5}$, Ross $C$ Hardison $^{6}$ and James Taylor ${ }^{1,2^{*}}$

\begin{abstract}
Background: Because species-specific gene expression is driven by species-specific regulation, understanding the relationship between sequence and function of the regulatory regions in different species will help elucidate how differences among species arise. Despite active experimental and computational research, relationships among sequence, conservation, and function are still poorly understood.

Results: We compared transcription factor occupied segments (TFos) for 116 human and 35 mouse TFs in 546 human and 125 mouse cell types and tissues from the Human and the Mouse ENCODE projects. We based the map between human and mouse TFos on a one-to-one nucleotide cross-species mapper, bnMapper, that utilizes whole genome alignments (WGA).

Our analysis shows that TFos are under evolutionary constraint, but a substantial portion ( $25.1 \%$ of mouse and $25.85 \%$ of human on average) of the TFos does not have a homologous sequence on the other species; this portion varies among cell types and TFs. Furthermore, $47.67 \%$ and $57.01 \%$ of the homologous TFos sequence shows binding activity on the other species for human and mouse respectively. However, $79.87 \%$ and $69.22 \%$ is repurposed such that it binds the same TF in different cells or different TFs in the same cells. Remarkably, within the set of repurposed TFos, the corresponding genome regions in the other species are preferred locations of novel TFos. These events suggest exaptation of some functional regulatory sequences into new function.

Despite TFos repurposing, we did not find substantial changes in their predicted target genes, suggesting that CRMs buffer evolutionary events allowing little or no change in the TFos - target gene associations. Thus, the small portion of TFos with strictly conserved occupancy underestimates the degree of conservation of regulatory interactions.

Conclusion: We mapped regulatory sequences from an extensive number of TFs and cell types between human and mouse using WGA. A comparative analysis of this correspondence unveiled the extent of the shared regulatory sequence across TFs and cell types under study. Importantly, a large part of the shared regulatory sequence is repurposed on the other species. This sequence, fueled by turnover events, provides a strong case for exaptation in regulatory elements.
\end{abstract}

Keywords: Mouse ENCODE, Regulatory sequences, Comparative genomics

\footnotetext{
*Correspondence: james@taylorlab.org

'Department of Mathematics and Computer Science, Emory University,

Atlanta, GA 30322, USA

2Department of Biology, Johns Hopkins University, 3400 N Charles St, Mudd

Hall 144, Baltimore, MD 21218, USA

Full list of author information is available at the end of the article
} 


\section{Background}

Most eukaryotic gene regulation occurs at the level of transcription [1,2]. This form of regulation involves the interaction of transcription factors (TFs) with element and function specific DNA sequences, referred to as cisregulatory modules (CRMs; reviewed by [3]). Their modular organization allows for elaborate regulatory mechanisms and fine control of gene expression [4].

Evolutionary changes in CRMs have a profound effect on species divergence. Many studies suggest that species specific CRMs are the defining factor for species identity [5-7]. Differences in human and chimpanzee, for example, are almost completely due to changes in functional noncoding sequence [8]. Efforts to locate the "human gene" have only revealed differences in a small number of genes [9-11]. Moreover, comparisons of organisms at the extremes of eukaryotes show that the genes encoding TFs and signaling components (e.g. for temporal/ spatial gene expression patterns) are largely conserved [4]. Taken together, this evidence suggests a hierarchical organization of regulatory networks. Modules at the top, performing essential upstream functions, span large evolutionary distances virtually unchanged, while lower level modules, involved in peripheral sub-networks, show a higher level of adaptation [12]. Under this model, part of the regulatory material must be under purifying selection and thus conserved between any two species of sufficiently small evolutionary divergence.

Evidence of conservation of regulatory sequence among species has inspired a series of computational methods. Some of these methods use machine learning and phylogenetic profiling to discover CRMs (reviewed by [13]), others use comparative analysis to identify genomic material under purifying evolutionary constraint as a representative of the functional genome (reviewed by [14]).

At the same time, a number of experimental studies suggest that while sequence might encode enough information to drive TF binding [15], the way this information is encoded is not trivial - similar sequence does not necessarily translate in similar function and vice versa. For example, regulatory elements have been found to tolerate sequence rearrangements [16] or even be under positive selection while maintaining the downstream regulatory machinery unchanged $[17,18]$. Recently, it was found that GATA1 changes its motif preferences during cell differentiation [19], serving as an example of TFs having multiple preferred motif sequences [20] while maintaining a regulatory function.

Both computational and experimental approaches have provided valuable insight into the regulatory portion of the genome, but they have limitations. Computational methods are biased toward well-annotated and evolutionarily conserved genomic regions, indeed comparative analyses based on evolutionary conservation alone ignore species-specific functional elements. Experimental approaches based on direct genome wide measurements of TFos are a powerful resource for the identification and analysis of TF binding sites. However, the number of cell types and TFs assessed so far has often been limited.

With some studies pointing at conservation, others at divergence, and others yet at turnover of motifs and the importance of occupancy, the level of constraint on the CRMs is still an open question. This apparently contradicting evidence can be reconciled by considering conservation as specific to the TF or the cell type.

In this study, we combined several types of functionassociated datasets from a large number of TFs from a wide variety of tissues and cell lines in both mouse and human. Our main data source is ChIP-Seq experiments performed by the mouse and human ENCODE projects. These data give evidence on the locations where TF have come close enough to the DNA to cross-link in cells. The question remains whether these locations, termed TFos [13], could represent direct binding to a specific motif in the DNA or co-association with another TF. However, there is evidence that the TFos are active in assays for regulatory function at a far higher rate than non-occupied DNA segments, or DNA segments predicted as regulatory based on sequence motifs or conservation making them likely to have regulatory function. We also used DNase I Hypersensitive Sites (DHS) generated by the Human [21] and the Mouse ENCODE [22] projects. DHS regions are markers of regulatory DNA and have underpinned the discovery of all classes of cis-regulatory elements including enhancers, promoters, insulators, silencers and locus control regions. We conduced a comparative analysis, by integrating TFos with human-mouse WGA, gene annotations, and TF-gene associations. We have also compiled the data and annotations derived from this study into a database to serve as a resource in exploring the relationship between sequence evolution and function of regulatory elements (Additional file 1: Figure S1).

\section{Results}

\section{An alignment based map for human-mouse TFos}

Differences between present day genomes are the result of a series of evolutionary events originating on their most recent common ancestor [23]. Many of these events can be explained under probability models and represented in the form of WGA (See [24] for a review). Sequence that has undergone a moderate number of evolutionary events will appear aligned, while highly divergent regions will usually be left un-aligned.

We used WGA to obtain a consistent map for TFos across human and mouse (Figure 1(A)). These alignments provide long, inferred homologous regions in the form of chains of gapless blocks. They can account for inversions, translocations, duplications, large-scale deletions, 


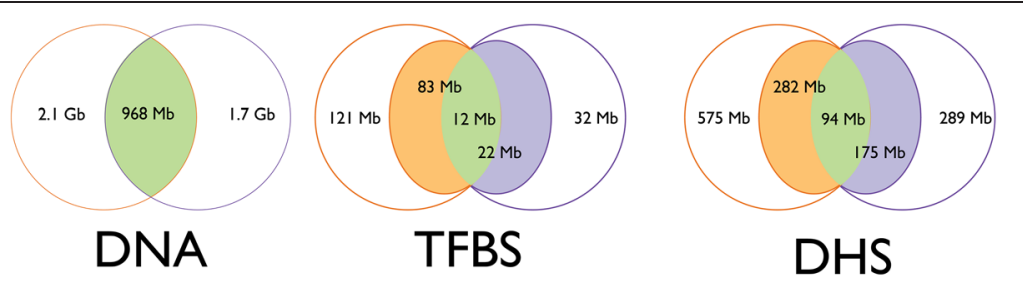

(A)

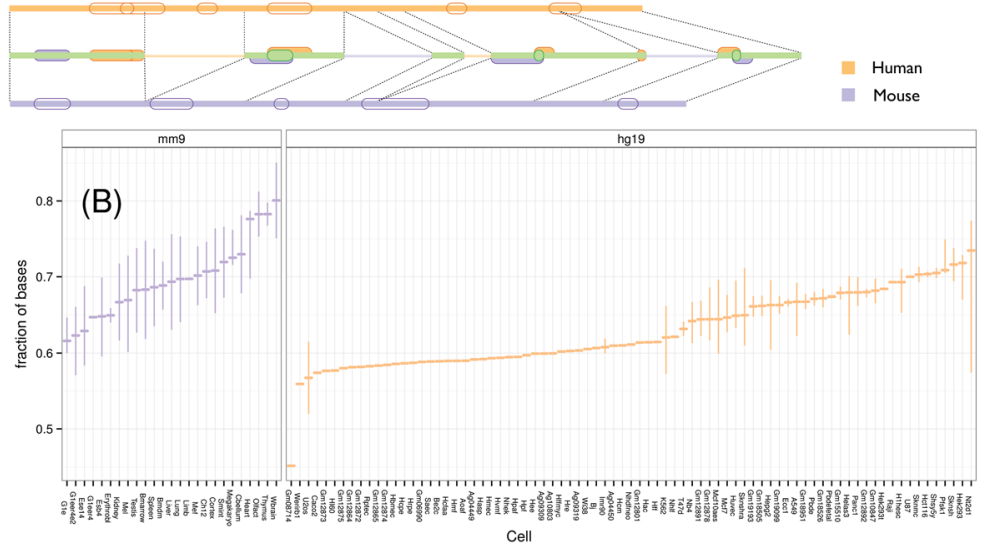

Figure 1 We built a one to one map between the human and mouse genomes from their WGA. (A) The track diagram shows TFos (unfilled features on outer tracks) and how they map to the other species (middle track). The thick part of the middle track is the mappable DNA. The features on the middle track can be mapped on the other species and are: SeqCons if not overlapping features from the other species (respective color), or FuncCons or FunctActive otherwise (green). The Venn diagrams use the same color code to show the amount (rounded to the closest Mb) of mappable material from one species to the other for DNA sequence, TFos, and DHS. For example, human TFos cover $121 \mathrm{Mb}$ of the human genome. When mapped to mouse, these TFos cover $83 \mathrm{Mb}$ of the mouse genome and $12 \mathrm{Mb}$ of the sequence covered also by mouse TFos. The diagram for DHS is labeled in a similar fashion. (B) The distribution of mappable TFos nucleotides across cell types. The box-plot for each cell type summarizes the distribution of values for the fraction of nucleotides covered by TFos that can be mapped on the other species.

and overlapping independent deletions. We considered chained blastz [25] alignments available from the UCSC browser [26] and the 12-way mammalian WGA from the EPO pipeline [27] available from Ensembl version 65 [28]. To choose the most appropriate alignment for our mappings we used several criteria: symmetry, coverage, feature enrichment, and other method specific properties.

Symmetry is important for unambiguously mapping features from one genome to the other and back. The EPO based map is inherently symmetric; we only need to extract human-mouse alignments. We remove segmental duplications based on criteria such as the score or the length of the alignment to obtain an unambiguous map. UCSC alignments, on the other hand, are based on blastz pairwise alignments, which are not symmetric - a humanmouse alignment is different from a mouse-human alignment in general. However, it is possible to circumvent the problem by using a netting procedure and chaining again only the first layer in the resulting net. This corresponds to heuristically cleaning overlapping chains based on their score. The resulting reciprocal map is identical to the one derived from the mouse-human alignments at the cost of losing around 10\% sequence coverage on both species [29].
UCSC alignments align a slightly larger fraction of the mouse genome ( $31 \%$ vs. $28 \%$ for UCSC and EPO respectively) to human. However, EPO alignments can assign a substantially higher amount of inserted mouse sequence ( $28 \%$ vs. $1.9 \%$ ). In total $65.7 \%$ of the mouse genome remains unmapped by UCSC alignments and $42.5 \%$ by EPO alignments.

Next, we computed the number of features that each alignment could map on the other species for all available cell types in human and mouse. We found that UCSC alignments mapped more features on the other species (Additional file 1: Figure S2 and Tables S4-S6).

Based on the above considerations, we adopted the reciprocal UCSC alignments. The alignments and the comparative pipeline described here were adopted by the Mouse Encode Consortium for the cross-species mapping of TFos.

\section{Function and sequence conservation of TFos}

We processed TFos generated by ChIP-Seq for $206 \mathrm{hu}-$ man and 55 mouse cell lines and tissues [22], with a variable number of factors for each cell or tissue (from 1 to 109 for human and from 1 to 38 for mouse). The data were generated by the Human and the Mouse ENCODE 
projects. The ChIP-Seq experiments on mouse were conducted following the human ENCODE guidelines [30]. Data processing, including Irreproducible Discovery Rate (IDR) analysis, was done using a uniform data processing pipeline for both datasets.

Elements recovered by the ChIP-Seq experiments and the subsequent peak-calling pipeline were subjected to thresholds for False Discovery Rate at 1\% and IDR at 2\%. We then filtered these sets to retain only those TFos showing DHS enrichment, thereby increasing our confidence in the functional role of the filtered elements. Roughly $41.63 \%$ of ChIP-Seq peaks were filtered out from each species by DHS filtering. The resulting data contained 5330864 and 727680 elements covering $121.08 \mathrm{Mb}$ and $31.6 \mathrm{Mb}$ of the genome for human and mouse respectively (Additional file 1: Table S3). The higher human coverage is due to the larger number of assays available in human.

We asked whether the selected putative regulatory material is significantly conserved. At the sequence level, we find that the fraction of TFos intersecting homologous regions is higher than one would expect by chance (Binomial test $0.99 \%$ CI: $(0.7218,0.7228)$ and $0.99 \% \mathrm{CI}$ : $(0.6908,0.6936)$ with expected values of 0.3129 and 0.3648 for human and mouse respectively). At the TF-binding activity level (i.e., having TF occupancy by any TF), mapped human and mouse TFos overlapped TFos on the other species more often than expected by chance (Binomial test 0.99\% CI: $(0.5358,0.5371)$ and $0.99 \%$ CI: $(0.7883,0.7913)$ with expected values 0.0231 and 0.0857 for human and mouse respectively). Significant conservation at the TF-binding activity level continues to hold largely for individual TFs-cell pairs (Additional file 1: Figures S3-S4). These results indicate that most of these human and mouse regulatory regions have been under selective pressure both at the sequence and functional level.

Despite the selective pressure on the TFos, the data suggest extensive evolution of the regulatory material between human and mouse. On average $74.2 \%$ and $74.9 \%$ of TFos can be mapped on the other species resulting in a $36.1 \%$ and $31.4 \%$ of regulatory sequence coverage that is lineage-specific for human and mouse respectively (Figure 1(B) and Additional file 1: S5-S12). Importantly, we found that the extent to which the remaining regulatory material is conserved varies substantially between species and among cell types and TFs. The variability fits with previous studies on smaller numbers of TFs and cell types, which have emphasized both, conservation of TFos [31] and extensive regulatory sequence evolution [32].

\section{Binding signal differences between functionally and sequence conserved TFos}

We focus our TFos comparative analysis on two Tier 1 ENCODE cell lines and their mouse analogs as chosen by the Mouse ENCODE consortium. The data consist of
17 TFs from human chronic myelogenous leukemia cell line (K562, [33]) and mouse erythroleukemia cell line (MEL, [34]); and 15 TFs from lymphoblast cell lines (GM12878 vs. CH12) (Additional file 1: Table S2). In total we have thus selected 442527 and 215251 TFos covering $33.61 \mathrm{Mb}$ and $18.77 \mathrm{Mb}$ on human and mouse respectively. We mapped TFos of a species to homologous locations in the other and asked what function do those sites show.

The homologous site of a TFos can be: (i) not occupied by TFs, in which case we call the TFos SeqCons; (ii) repurposed, thus active in another cell or bound by another factor in the same cell type, in which case we call the TFos FunctActive; or (iii) bound by the same factor on the same cell type, in which case the TFos is called FunctCons. SeqCons and FunctActive elements represent differences in human and mouse TF binding patterns due to TFos loss, gain, or both. Turnover occurs in the case of loss and gain of the same TFos at different, but nearby, positions. However, more complex differences can arise when the loss of one TFos is followed by compensatory gains of TFos of other TFs. For the pairs of analogous cell types in human and mouse, the largest proportion of TFos (considered together) are FunctActive, while the relative proportions of FunctCons and SeqCons vary between cell types and species (Figure 2(A)).

Conservation of occupancy in the other species was associated with peak signal strength for several cell typeTF pairs. For example, binding signal on FunctCons or FunctActive elements was higher than that on SeqCons elements for $59 \%$ and $50 \%$ of cell type-TF combinations for human and mouse respectively (with a Bonferronicorrected error rate of 1\%; Additional file 1: Figures S13, S14). Overall, FunctCons peak signal is on average 1.3 and 1.9 times larger than SeqCons peak signal on human and mouse respectively (Additional file 1: Table S1).

\section{TF binding site turnover and TFos repurposing}

If SeqCons and FunctActive elements are the result of turnover of TF binding sites (TFBS) in human and mouse, then we should observe TFBS close to regions orthologous to TFBS on the other species. We discovered TFBS using motif discovery and motif matching on TFos for both species and analyzed their alignment. We found that for several TFs, SeqCons TFBS map within 150 bp of a TFBS on the other species. On average, $51 \%$ and $48 \%$ of SeqCons TFBS have been subject to turnover in a 150 bp neighborhood for human and mouse respectively (Figure 2(B)).

The large number of FunctActive elements (Figures 2(A) and Additional file 1: S15, S16) suggests recycling of TFos among cells and TFs. We examined this more carefully with a novel approach, based on the intuition that extensive recycling would allow any set of TFos in a comparison species to identify the occurrence of specific TFos in the 

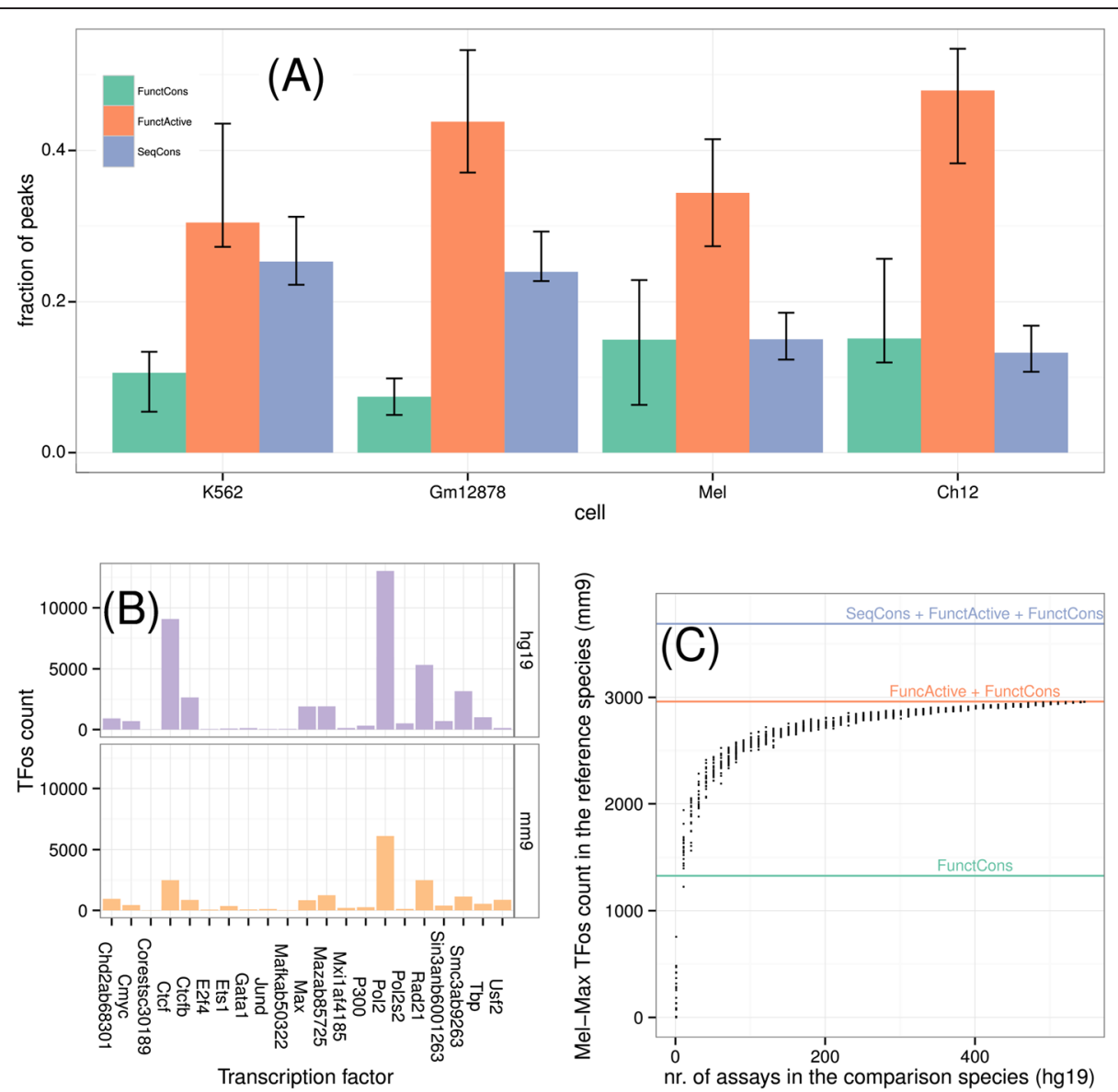

Figure 2 Conservation and re-use of TFos. (A) The distribution of SeqCons, FunctActive, and FunctCons regulatory elements summarized for all human mouse analogous cell lines (Additional file 1: Table S2). In both cell lines, a small fraction of mappable regulatory elements is FunctCons (33\% and 15\% cell average for human and mouse respectively). A larger fraction (46\% and 50\% cell average for human and mouse respectively) is FunctActive (plots for each cell are in Additional file 1: Figures S15, S16). (B) Loss and gain of TF binding sites. We used TFos in our data to discover TF binding sites (TFBS) on both species. We mapped human (respectively mouse) TFBS to mouse (respectively human) and computed their distance to the closest mouse (respectively human) TFBS of the same TF on an analogous cell. Among these TFBS those with a positive distance but less than $150 \mathrm{bp}$ contribute to the corresponding bar in the human (respectively mouse) subplot. In other words, each bar is the count of TFBS that were lost and gained within a $150 \mathrm{bp}$ window of the original site. (C) Reuse of FunctActive TFos between different cells and factors. A point on the scatterplot indicates the number of TFos of the corresponding assay in the reference species that can be classified as FunctCons or FunctActive when considering a fixed size set of randomly chosen assays in the comparison species. We performed multiple computations for each chosen size of such sets. Lines indicate the accumulated number of FunctCons, FunctActive, and Seqcons TFos. In this figure, about 93\% of the (Mel, Max) assay is covered by TFos from just 35\% of the query assays (plots for other cells/TFs in Additional file 1: Figures S17, S18).

reference species (after cross-species mapping). To conduct this analysis, we sampled (multiple times) mapped TFos from $k$ assays from the comparison species, took the union of these TFos, and computed the number of TFos a given assay (in the reference species) shares with this union. The results strongly support extensive recycling of TFos. Specifically, we find that about $93 \%$ of the (Mel, Max) assay is covered by TFos from just 35\% of the query assays. As $k$ increases, coverage saturates (Figures 2(C) and Additional file 1: S17, S18). This ability of different assays in the comparison species to capture TFos in the reference species is substantially different than what is obtained by simulating non-associated assays (Additional file 1: Figure S19).

We tested the hypothesis that new TFos tend to arise over existing TFos - active in other cells or occupied by other factors. Under the null hypothesis, the fraction of FunctActive elements with respect to the non-FunctCons elements should not be significantly higher than the fraction of the genome that can turn into a FunctActive TFos, should a new TFos occur (See Methods for details). We found that fraction to be higher than expected (One sided Binomial test $99 \%$ CI: $(0.377,1.000)$ and $(0.477,1.000)$ with expected values 0.010 and 0.028 for human and 
mouse respectively), suggesting that existing CRMs are sites where new TFBS are likely to arise.

Turnover events or the appearance of novel TFBS lead to compositional changes in CRMs. However, these changes do not always reflect downstream in, for example, the set of target genes. Following [35] we extracted a set of TSS-enhancer connections based on synchronized DHS activity during the transcription of a gene. Restricting on 15,736 human-mouse orthologous genes [22], we were thus able to define putative target genes for 1928211 and 204758 TFos for human and mouse respectively. We observe that, similarly to FunctCons TFos, about 39\% and $43 \%$ of FunctActive TFos retained at least one putative target gene for human and mouse respectively. Furthermore, the amount at which the set of target genes is conserved across species is not significantly different between the two classes of TFos (Kolmogorov-Smirnov test. p-values: $<2.2 \mathrm{e}-16$ and $<2.2 \mathrm{e}-16$ for human and mouse respectively). The example of the candidate enhancer linked to the ACAP3 gene in human illustrates this situation (Figure 3). The DNA segment in mouse that is orthologous to the human enhancer has been repurposed such that it binds different TFs, but the mouse Acap3 gene is still the presumptive target.

\section{Discussion}

We reported on the construction and use of a map of functional elements between human and mouse based on WGA. This map is consistent and symmetrical in that it provides a one to one correspondence between genome elements in both directions. Furthermore, it constitutes an improvement over aligning TFos directly on the other species guided by gene orthology; this approach is likely to deteriorate with the distance of an element from the nearest gene with an ortholog on the other species.
Using this mapping approach, we were able to recover, on average, $75 \%$ and $73 \%$ of TFos in the other species, of which $63 \%$ and $78 \%$ are bound by any TFs and $13 \%$ and $25 \%$ by the same TF for human and mouse respectively. The rest of the regulatory material is species-specific either at the sequence or functional level and is likely to account for the phenotypic differences between human and mouse $[8,12]$. However, some of the species-specific material retains target genes suggesting that CRMs buffer changes toward downstream regulation [36].

One potential difficulty, which affects this analysis, is the variation that can be introduced by technical factors such as signal thresholding or by differences in the environment of the cells being compared. By using data processed for reproducibility under the stringent standards developed by the Human and Mouse ENCODE projects, restricting to regions overlapping DHS for greater confidence, considering the TF activity on analogous cell types, and employing statistical controls we hope to have ameliorated these issues.

Previous studies have mapped the binding patterns of TFs between species, including mouse and human, by focusing on a single cell type and a few TFs (e.g., [32]). While these important studies have revealed substantial divergence in the binding patterns of TFs between species, they do not explore FunctActive elements or related dynamics such as repurposing. One important conclusion in our study is that the evolution of regulatory sequences varies considerably among TFs and cell types, and furthermore we document which TFos fall into the various evolutionary categories.

The traffic between noncoding functional and nonfunctional DNA in a genome is two-way. On the one hand, loss of a TFos can increase fitness, as dramatically illustrated by changes in the regulation of pitx 1 in three-spined

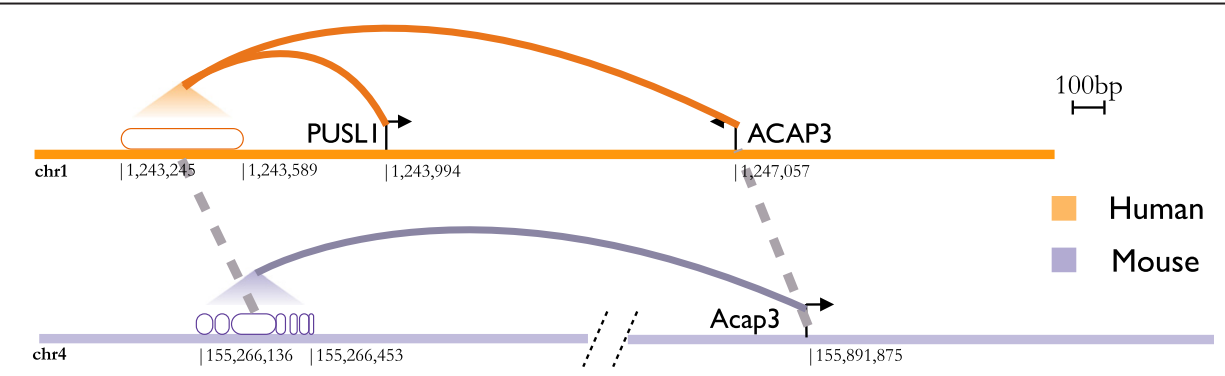

Figure 3 Conservation of presumptive gene targets for a repurposed TFos. We combined cross-species gene-gene and TFos-TFos associations and gene-TFos associations to determine whether the sets of target genes of FunctCons and FunctActive TFos differ significantly. Gene-gene association data are based on a set of orthologous genes between human and mouse produced by the mouse ENCODE consortium, gene-TFos data are based on synchronized DHS activity during gene transcription [35], and TFos-TFos associations are based on our cross-species map. In the figure, we show a human TFos of Mxi on K562 (empty oval) associated with PUSL1 and ACAP3. This TFos is FunctActive, since its analogous location (broken oval linked by the dashed line with spaces between ovals indicating insertions in mouse) in mouse is bound by other TFs on other cell types (not shown). However, its analogous site in mouse is linked to gene Acap3. Incidentally, ACAP3 and Acap3 are orthologous and in our gene-gene association set. The human TFos and its analogous site in mouse bind different TFs and are active in different cell types, but they share a target gene. 
stickleback that lead to advantageous anatomical changes in a new environment. On the other hand, new functional noncoding sequence can arise from nonfunctional sequence through turnover [37]. The differences between the sizes of FunctActive and FunctCons pools suggests another source of functional noncoding material traffic, characterized by the exaptation of TFos into functional noncoding material with novel TF or cell type activity [38]. With a partial catalog of regulatory elements it is hard to distinguish the newly created functional material from the exapted one. However with ever-increasing numbers of experimental assays the picture should become more clear.

\section{Conclusions}

Despite previous elegant studies, the level of constraint on CRMs remains controversial. We provide further evidence that TFos are more conserved than random sequence and that conservation is cell and TF specific. Furthermore, we study the type of conservation of TFos and observe that $47.67 \%$ and $57.01 \%$ of TFos conserved at the sequence level for human and mouse respectively have been repurposed and are active in cell types or bind different TFs. We show how this sequence makes a case for TFos exaptation into new function. Finally, we find that repurposing does not necessarily lead to changes in the TFos - target gene association.

Comparative studies of CRMs involve a mapping strategy of regulatory elements, often by aligning elements or reads on both genomes, possibly guiding mapping with gene homologies. We implemented a multiple WGA based process for TFos mapping. Using multiple WGA we should have a more sensitive and consistent mapping process. Importantly, the mapper can provide one-toone mappings between species, which is very useful during analysis.

\section{Methods}

\section{Data processing}

\section{ChIP-Seq data}

We download all of the ChIP-Seq data generated by the Human and the Mouse ENCODE from the ENCODE DCC. The pipeline that filters the original ENCODE peaks is available in the supplementary material.

\section{Distal DHS-to-promoter connections}

As described in [35] many cell-selective enhancers become DHSs synchronously with the appearance of hypersensitivity at the promoter of their target gene. This has been used to infer a genome-wide DHS/enhancer-promoter connection set. Using a conservative list of orthologous genes [22] we inferred a list of correspondences between TFos regulating human-mouse orthologous genes.

\section{EPO and UCSC based maps}

We considered the EPO 12-way mammalian whole genome alignments from Ensembl project version 65 [28] and the UCSC human mouse chain and net alignments from the UCSC [26] genome browser to generate the reciprocal maps. Details and programs for processing of the alignments are available at https://bitbucket.org/bxlab/ mapper_comparisons.

\section{Mapping strategy}

We built and used a one-to-one nucleotide mapper (bnMapper) to map elements between human and mouse. The mapping is bijective, so reverse application of the mapping to a mapped nucleotide, returns the original nucleotide. Elements that span matching blocks of different chains and elements that map to multiple chromosomes are filtered out. The mapper and a detailed analysis of performance between EPO and UCSC alignments are available at https://bitbucket.org/bxlab/mapper_comparisons. Details on the mapping strategy are on Section 1.1 of the Additional file 1 .

\section{Significance tests}

For a given genomic region of coverage $C$, the number of $\mathrm{n}$ randomly positioned features over the genome (length $L$ ) would follow a Binomial $(n, C / L)$. For significance of TF conservation at the sequence level we set $L, C$, and $n$, to be the length of the genome, total TF coverage, and total number of peaks. A success event is an overlap of $1 \mathrm{bp}$ with the one to one mappable sequence. Similarly, for functional conservation, $L, C$, and $n$, are set to total one to one mappable sequence, coverage of mapped peaks, and number of mapped peaks overlapping with peaks on the target genome, respectively.

For the paired Wilcoxon tests we consider all TFs within a cell line that appear on both species. Ranks are computed from FunctCons to SeqCons ratios of corresponding TFs on each species.

To test that a new TFos is more likely to occur in an existing TFos, possibly occupied by another TFs or active in another cell, consider the following quantities with respect to a fixed genome:

- The number of non-FunctCons TFos in this genome not less than the number of new TFos in this genome. The inequality accounts for TFos deletions on the other genome. We write $n F C o>=n F C t$.

- Coverage of SeqCons of the other genome after being mapped in this genome and FA elements in this genome not less than regions in this genome that would become FA should a new TFos occur. The inequality accounts for those regions that would become FunctCons should a new TFos appear. We write $M o>=M t$. 
- Length of this genome. We write $L$.

- The number of FunctActive Tfos in this genome. We write $F A$.

Here we are making the assumption that FunctCons elements have existed before human-mouse split and FunctActive are a result of a deletion or creation (or both) after the human-mouse split. Thus, the fraction $F A / n F C t$ is that of new elements that occurred in existing TFos involving other TFs or cell types, whether the fraction $M t / L$ indicates the chance of a new TFos to become FunctActive should it occur randomly in the genome. Under the alternative hypothesis, $F A / n F C t>M t / L$. However, because $M t / L<=M o / L$ and $F A / n F C o<=F A$ / $n F C t$, it suffices to show that $F A / n F C o>=M o / L$. Notice that we observe all the quantities in the last expression.

\section{Data access}

UCSC alignments can be downloaded from the UCSC website http://hgdownload.soe.ucsc.edu/goldenPath/hg19/ vsMm9/.

UCSC bijective (netted) alignments, the list of one-toone orthologous genes, the list of DHS-gene associations, and the list of Mouse ChIP-Seq TFos and DHSs can be downloaded from the Mouse ENCODE web portal http://mouse.encodedcc.org/data and http://www. mouseencode.org/.

The mapping software can be freely downloaded as part of the bx-python software library from https://bitbucket. org/james_taylor/bx-python/wiki/Home.

Ensembl EPO 12-way alignments version 65 can be downloaded from the Ensembl (December 2011) at ftp:// ftp.ensembl.org/pub/release-65/emf/ensembl-compara/epo 12_eutherian/ and http://www.ebi.ac.uk/ kbeal/species_ mapper/epo_547_hs_mm_12way_mammals_65.out.gz.

The human ChIP-Seq TFos and DHSs can be downloaded from the Human ENCODE website at http://genome.ucsc. edu/ENCODE/.

\section{Additional file}

Additional file 1: Supplemental Figures and Tables.

\section{Competing interests}

The authors declare that they have no competing interests.

\section{Authors' contributions}

OD wrote the main text, performed the comparative and statistical analysis, and wrote the mapper tool. RS helped designing the mapper, carried out statistical analysis for choosing the best whole genome alignments, prepared the DHS data, and the gene - TFos link data. YC helped on the design of the mapper and performed analyses on the whole genome alignments. KB worked on the design of the mapper and the preparation of the whole genome alignments. JH worked on the design of the mapper and the preparation of the whole genome alignments, helped design the study and write the main text. RC wrote the main text and designed the study - JT wrote the main text, carried out statistical analyses, and designed the study. All authors read and approved the final manuscript.

\section{Acknowledgments}

Thanks to members of the Taylor and Hardison labs and the Mouse ENCODE consortium for their input and discussion. This project was supported by grants from the National Institutes of Health to RCH and JT, specifically American Recovery and Reinvestment Act (ARRA) funds through grant number RC2 HG005573 from the National Human Genome Research Institute, and grants with numbers R01 DK065806 and R56 DK065806 from the National Institute for Diabetes, Digestive, and Kidney Diseases. This work was also supported by the Wellcome Trust (grant number 095908) and the European Molecular Biology Laboratory. The funding body had no role in the design of the experiment, in the collection, analysis, and interpretation of data, in the writing of the manuscript, or in the decision to submit the manuscript for publication.

\section{Author details}

'Department of Mathematics and Computer Science, Emory University, Atlanta, GA 30322, USA. ²Department of Biology, Johns Hopkins University, 3400 N Charles St, Mudd Hall 144, Baltimore, MD 21218, USA. Department of Genetics, Stanford University, Stanford, CA 94305, USA. ${ }^{4}$ Department of Genome Sciences, University of Washington, Seattle, WA 98195, USA. ${ }^{5}$ The Genome Analysis Centre, Norwich Research Park, Norwich, UK. ${ }^{6}$ Department of Biochemistry and Molecular Biology, Penn State University, University Park, Norwich, PA 16802, USA. 'European Bioinformatics Institute (EMBL-EBI), Norwich, UK

Received: 8 October 2014 Accepted: 15 January 2015

Published online: 14 February 2015

\section{References}

1. Derman E, Krauter K, Walling L, Weinberger C, Ray M, Darnell Jr JE. Transcriptional control in the production of liver-specific mRNAs. Cell. 1981;23(3):731-9.

2. Roop DR, Nordstrom JL, Tsai SY, Tsai MJ, O'Malley BW. Transcription of structural and intervening sequences in the ovalbumin gene and identification of potential ovalbumin mRNA precursors. Cell. 1978;15(2):671-85.

3. Maston GA, Evans SK, Green MR. Transcriptional regulatory elements in the human genome. Annu Rev Genomics Hum Genet. 2006;7:29-59.

4. Davidson $\mathrm{EH}$. The regulatory genome: gene regulatory networks in development and evolution. Burlington (MA): Academic Press; 2006.

5. Meader S, Ponting CP, Lunter G. Massive turnover of functional sequence in human and other mammalian genomes. Genome Res. 2010;20(10):1335-43.

6. Taft RJ, Pheasant M, Mattick JS. The relationship between non-proteincoding DNA and eukaryotic complexity. Bioessays. 2007;29(3):288-99.

7. Britten RJ, Davidson EH. Gene regulation for higher cells: a theory. Science. 1969;165(3891):349-57

8. King MC, Wilson AC. Evolution at two levels in humans and chimpanzees. Science. 1975;188(4184):107-16.

9. Hughes AL, Yeager M. Natural selection at major histocompatibility complex loci of vertebrates. Annu Rev Genet. 1998:32:415-35.

10. Swanson WJ, Yang Z, Wolfner MF, Aquadro CF. Positive Darwinian selection drives the evolution of several female reproductive proteins in mammals. Proc Natl Acad Sci U S A. 2001;98(5):2509-14.

11. Enard W, Przeworski M, Fisher SE, Lai CS, Wiebe V, Kitano T, et al. Molecular evolution of FOXP2, a gene involved in speech and language. Nature. 2002;418(6900):869-72.

12. Davidson $\mathrm{EH}$, Erwin $\mathrm{DH}$. Gene regulatory networks and the evolution of animal body plans. Science. 2006;311(5762):796-800.

13. Hardison RC, Taylor J. Genomic approaches towards finding cis-regulatory modules in animals. Nat Rev Genet. 2012;13(7):469-83.

14. Ponting CP, Hardison RC. What fraction of the human genome is functional? Genome Res. 2011;21(11):1769-76.

15. Wilson MD, Barbosa-Morais NL, Schmidt D, Conboy CM, Vanes L, Tybulewicz $V L$, et al. Species-specific transcription in mice carrying human chromosome 21. Science. 2008:322(5900):434-8.

16. Hare EE, Peterson BK, lyer VN, Meier R, Eisen MB. Sepsid even-skipped enhancers are functionally conserved in Drosophila despite lack of sequence conservation. PLoS Genet. 2008;4(6):e1000106. 
17. Pheasant M, Mattick JS. Raising the estimate of functional human sequences. Genome Res. 2007;17(9):1245-53.

18. Bustamante CD, Fledel-Alon A, Williamson S, Nielsen R, Hubisz MT, Glanowski S, et al. Natural selection on protein-coding genes in the human genome. Nature. 2005;437(7062):1153-7.

19. May G, Soneji S, Tipping AJ, Teles J, McGowan SJ, Wu M, et al. Dynamic analysis of gene expression and genome-wide transcription factor binding during lineage specification of multipotent progenitors. Cell Stem Cell. 2013;13(6):754-68.

20. Johnson DS, Mortazavi A, Myers RM, Wold B. Genome-wide mapping of in vivo protein-DNA interactions. Science. 2007:316(5830):1497-502.

21. The ENCODE Project Consortium, Bernstein BE, Birney E, Dunham I, Green ED, Gunter C, et al. An integrated encyclopedia of DNA elements in the human genome. Nature. 2012;489((7414):57-74.

22. The Mouse ENCODE Consortium, Yue F, Cheng Y, Breschi A, Vierstra J, Wu W, et al. A comparative encyclopedia of DNA elements in the mouse genome. Nature 2014:515(7527):355-64

23. Kimura M. Evolutionary rate at the molecular level. Nature. 1968;217(5129):624-6.

24. Ureta-Vidal A, Ettwiller L, Birney E. Comparative genomics: genome-wide analysis in metazoan eukaryotes. Nat Rev Genet. 2003;4(4):251-62.

25. Schwartz S, Kent WJ, Smit A, Zhang Z, Baertsch R, Hardison RC, et al. Human-mouse alignments with BLASTZ. Genome Res. 2003;13(1):103-7.

26. Dreszer TR, Karolchik D, Zweig AS, Hinrichs AS, Raney BJ, Kuhn RM, et al. The UCSC genome browser database: extensions and updates 2011. Nucleic Acids Res. 2012;40(Database issue):D918-23.

27. Paten B, Herrero J, Beal K, Fitzgerald S, Birney E. Enredo and Pecan: genome-wide mammalian consistency-based multiple alignment with paralogs. Genome Res. 2008;18(11):1814-28.

28. Flicek P, Ahmed I, Amode MR, Barrell D, Beal K, Brent S, et al. Ensembl 2013. Nucleic Acids Res. 2013;41(Database issue):D48-55.

29. Kent WJ, Baertsch R, Hinrichs A, Miller W, Haussler D. Evolution's cauldron: duplication, deletion, and rearrangement in the mouse and human genomes. Proc Natl Acad Sci U S A. 2003;100(20):11484-9.

30. Landt SG, Marinov GK, Kundaje A, Kheradpour P, Pauli F, Batzoglou S, et al. ChIP-seq guidelines and practices of the ENCODE and modENCODE consortia. Genome Res. 2012;22(9):1813-31.

31. Kim TH, Abdullaev ZK, Smith AD, Ching KA, Loukinov DI, Green RD, et al. Analysis of the vertebrate insulator protein CTCF-binding sites in the human genome. Cell. 2007;128(6):1231-45.

32. Odom DT, Dowell RD, Jacobsen ES, Gordon W, Danford TW, Maclsaac KD, et al. Tissue-specific transcriptional regulation has diverged significantly between human and mouse. Nat Genet. 2007:39(6):730-2.

33. Lozzio CB, Lozzio BB. Human chronic myelogenous leukemia cell-line with positive Philadelphia chromosome. Blood. 1975;45(3):321-34.

34. The Mouse ENCODE Consortium, Stamatoyannopoulos JA, Snyder M, Hardison R, Ren B, Gingeras T, et al. An encyclopedia of mouse DNA elements (Mouse ENCODE). Genome Biol. 2012;13(8):418.

35. Thurman RE, Rynes E, Humbert R, Vierstra J, Maurano MT, Haugen E, et al. The accessible chromatin landscape of the human genome. Nature. 2012;489(7414):75-82.

36. Weirauch MT, Hughes TR. Conserved expression without conserved regulatory sequence: the more things change, the more they stay the same. Trends Genet. 2010;26(2):66-74.

37. Wilson MD, Odom DT. Evolution of transcriptional control in mammals. Curr Opin Genet Dev. 2009;19(6):579-85.

38. Gould SJ, Vrba ES. Exaptation - a missing term in the science of form. Paleobiology. 1982:8(1):4-15.

\section{Submit your next manuscript to BioMed Central and take full advantage of:}

- Convenient online submission

- Thorough peer review

- No space constraints or color figure charges

- Immediate publication on acceptance

- Inclusion in PubMed, CAS, Scopus and Google Scholar

- Research which is freely available for redistribution

Submit your manuscript at www.biomedcentral.com/submit 